\title{
OPTIMAL CONTROL OF PDEs WITH REGULARIZED POINTWISE STATE CONSTRAINTS
}

\author{
Christian Meyer, Arnd Rösch, Fredi Tröltzsch
}

Preprint 2003/14

Preprint-Reihe des Instituts für Mathematik Technische Universität Berlin 
This paper addresses the regularization of pointwise state constraints in optimal control problems. By analyzing the associated dual problem, it is shown that the regularized problems admit Lagrange multipliers in $L^{2}$-spaces. Under a certain boundedness assumption, the solution of the regularized problem converges to the one of the original state constrained problem. The results of our analysis are confirmed by numerical tests.

AMS subject classifications. 49K20, 49N10, 90C46, 49M20 


\title{
OPTIMAL CONTROL OF PDES WITH REGULARIZED POINTWISE STATE CONSTRAINTS *
}

\author{
CHRISTIAN MEYER, ARND RÖSCH, FREDI TRÖLTZSCH ${ }^{1}$
}

\begin{abstract}
This paper addresses the regularization of pointwise state constraints in optimal control problems. By analyzing the associated dual problem, it is shown that the regularized problems admit Lagrange multipliers in $L^{2}$-spaces. Under a certain boundedness assumption, the solution of the regularized problem converges to the one of the original state constrained problem. The results of our analysis are confirmed by numerical tests.
\end{abstract}

Key words. Quadratic programming, regular Lagrange multipliers, optimal control, elliptic and parabolic equations, pointwise state constraints, bottleneck constraints.

AMS subject classifications. 49K20, 49N10, 90C46, 49M20

1. Introduction. In this paper, we suggest a regularization method to solve certain classes of optimal control problems with pointwise state constraints of the form $y(x) \geq y_{c}(x)$ in a certain subset $D \subset \mathbb{R}^{N}$, where $y$ is the solution of a partial differential equation associated with a control function $u \in L^{2}(D)$. If $y$ and $y_{c}$ can be assumed to be continuous, then the Lagrange multipliers associated with the state constraints are measures, see, for instance, Casas [6] or Raymond [9] for the case of elliptic and parabolic control problems. Therefore, the numerical treatment of such problems is difficult. Moreover active-set strategies such as the ones proposed by Bergounioux, Ito and Kunisch [2], Bergounioux and Kunisch [3] or Kunisch and Rösch [8] cannot directly be applied to constraints of the form $y(x) \geq y_{c}(x)$. The situation changes, if mixed pointwise control-state constraints are given, say $u(x) \leq y_{c}(x)+y(x)$ or $-u(x) \leq y_{c}(x)+y(x)$. Although these constraints must be considered in $L^{2}(D)$, if $u \in L^{2}(D)$, special techniques apply to prove the existence of regular Lagrange multipliers in $L^{2}(D)$. We refer to Bergounioux and Tröltzsch [4], [5] or Arada and Raymond [1] or Casas, Raymond and Zidani [7] for parabolic control problems. This experience led us to regularize the pointwise state constraints by

$$
y(x) \geq y_{c}(x)-\varepsilon u(x)
$$

where $\varepsilon>0$ is a small parameter. This paper is to show that this idea exhibits a satisfactory numerical performance. We show the strong convergence of optimal contols for $\varepsilon \downarrow 0$ and verify the analysis by associated numerical tests. Before defining a quite general class of optimization problems in Hilbert spaces that covers in particular elliptic and parabolic problems, let us explain our approach by an exemplary situation.

2. An introductory example for constraint regularization. We consider the following elliptic control problem with pointwise state constraints and distributed

\footnotetext{
*Supported by the DFG Research Center "Mathematics for key technologies" (FZT 86) in Berlin.

${ }^{1}$ Institut für Mathematik, Technische Universität Berlin, D-10623 Berlin, Str. des 17. Juni 136, Germany.
} 
control

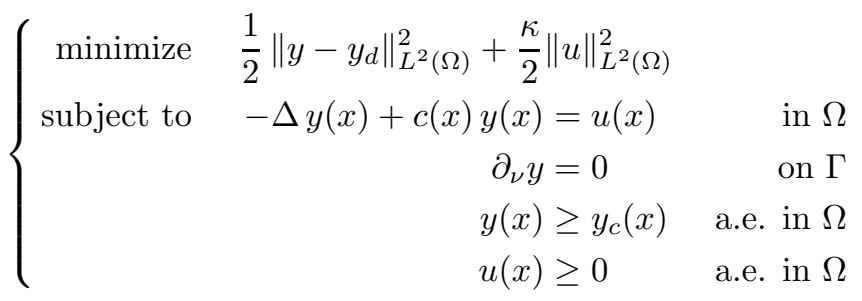

where $\Omega \subset \mathbb{R}^{N}, N \geq 2$, is a bounded domain with $C^{0,1}$-boundary $\Gamma$, $\kappa$ is a fixed positive real number and $y_{d}$ and $y_{c}$ are given functions from $L^{2}(\Omega)$. Furthermore, $c(x) \geq 0$ is a fixed function from $L^{\infty}(\Omega)$ with $c(x)>0$ on a measurable subset of $\Omega$. By $\partial_{\nu}$ the normal derivative with respect to the outward normal vector is denoted.

It is well known that the Lagrange multipliers associated with pointwise state constraints can be Borel measures on $\Omega$. They need not be measurable functions on $\Omega$, see for instance [6]. However, the situation is different, if mixed control-state constraints of the form $y \geq y_{c}-\varepsilon u$ are given. Therefore, we introduce an optimization problem with regularized state constraints, where, under natural assumptions, the Lagrange multipliers can assumed to be functions from $L^{2}(\Omega)$. This modified optimization problem is

$$
\left(\mathrm{P} 1_{\varepsilon}\right) \quad\left\{\begin{array}{rlrl}
\text { minimize } & \frac{1}{2}\left\|y-y_{d}\right\|_{L^{2}(\Omega)}^{2}+\frac{\kappa}{2}\|u\|_{L^{2}(\Omega)}^{2} & \\
\text { subject to } & -\Delta y(x)+c(x) y(x) & =u(x) & \\
\partial_{\nu} y & =0 & \text { in } \Omega \\
y(x) & \geq y_{c}(x)-\varepsilon u(x) & & \text { a.e. in } \Omega \\
u(x) & \geq 0 & & \text { a.e. in } \Omega
\end{array}\right.
$$

with a regularization parameter $\varepsilon>0$ and $y_{c}, y_{d}, c$ and $\kappa$ as defined above.

This regularization can also be justified in another way: Let us suppose for a while that the state constraint at the optimal state is active everywhere, i.e. $y(x) \equiv y_{c}(x)$. The solution operator of the elliptic equation $S: u \mapsto y$ is compact, hence the associated equation for $u, S u=y_{c}$, is ill-posed. This gives another hint that the regularization to $\varepsilon u+S u=y_{c}$ should be useful. In this sense, our approach represents a Lavrentiev type regularization.

Within this paper we will show that problem $\left(\mathrm{P} 1_{\varepsilon}\right)$ has a regular Lagrange multiplier and a unique optimal solution from $L^{2}(\Omega)$ that strongly converges to the optimal solution $\bar{u}$ of the initial problem (P1) when $\varepsilon$ converges to zero.

We present our theory for a more general class of optimization problems that also covers $(\mathrm{P} 1)$ and $\left(\mathrm{P} 1_{\varepsilon}\right)$, respectively.

3. A general class of optimization problems with state constraints. We embed our introductory example into the more general optimization problem

$$
\left\{\begin{array}{rlrl}
\operatorname{minimize} & \frac{1}{2}\|S u\|_{H}^{2} & +\int_{D}\left(a(x) u(x)+\frac{\kappa}{2} u(x)^{2}\right) d x \\
\text { subject to } & (G u)(x) & \geq y_{c}(x) & \text { a.e. in } D \\
u(x) & \geq 0 & \text { a.e. in } D .
\end{array}\right.
$$


In this problem, $D \subset \mathbb{R}^{N}$ is a Lebesgue-measurable bounded set while $G$ and $S$ are linear compact operators from $L^{2}(D)$ to real Hilbert spaces $H$ and $L^{2}(D)$, respectively. The functions $y_{c}$ and $a$ are fixed in $L^{2}(D)$ and $\kappa>0$ is a fixed constant. Let us denote the natural inner product of $L^{2}(D)$ by $(\cdot, \cdot)$ and the associated norm by $\|\cdot\|$. In all what follows, we denote the primal objective function by $f$, i.e.

$$
f(u)=\frac{1}{2}\|S u\|_{H}^{2}+\frac{\kappa}{2}\|u\|^{2}+(a, u) .
$$

In the corresponding regularized problem, we again introduce a regularization term in the state constraint,

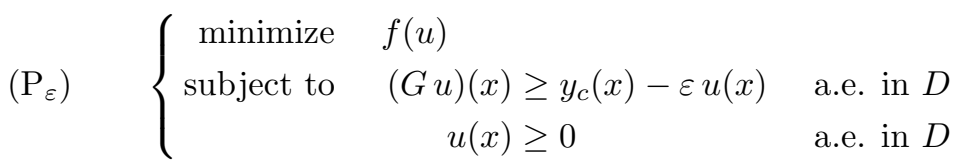

with some real number $\varepsilon>0$.

The problems $(\mathrm{P} 1)$ and $\left(\mathrm{P} 1_{\varepsilon}\right)$ are related to the general case as follows: In (P1) and $\left(\mathrm{P} 1_{\varepsilon}\right), D$ equals $\Omega$ and $H=L^{2}(\Omega)$. It is known that the elliptic Neumann problem given in $(\mathrm{P} 1)$ and $\left(\mathrm{P} 1_{\varepsilon}\right)$ admits for each $u \in L^{2}(\Omega)$ a unique weak solution $y \in$ $H^{1}(\Omega)$. Thanks to the compact embedding of $H^{1}(\Omega)$ in $L^{2}(\Omega)$, the solution operator $S: u \mapsto y$ can be regarded as a linear compact operator from $L^{2}(\Omega)$ to $L^{2}(\Omega)$. Thus, the operator $S$ in the general problem is the solution operator $S: L^{2}(\Omega) \rightarrow L^{2}(\Omega)$. Expanding the first norm square in the objective functional in $(\mathrm{P} 1)$ and $\left(\mathrm{P} 1_{\varepsilon}\right)$, we get $\frac{1}{2}\left\|y-y_{d}\right\|_{L^{2}(\Omega)}^{2}=\frac{1}{2}\|y\|_{L^{2}(\Omega)}^{2}-\left(S^{\star} y_{d}, u\right)+$ const., hence $a=-S^{\star} y_{d}$. As $y$ appears in the first constraint, the operator $G$ is defined by $G=S$.

A parabolic example. A second example is represented by the following parabolic boundary control problem:

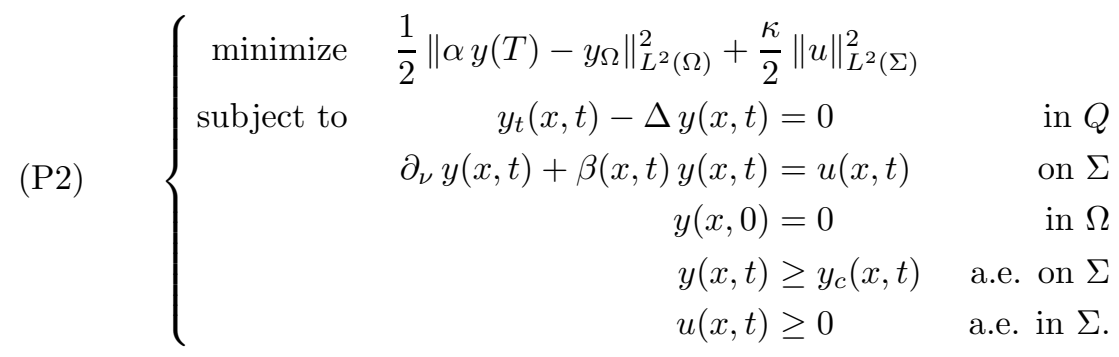

Here, the standard notations $Q=\Omega \times(0, T), \Sigma=\Gamma \times(0, T)$ are used. Moreover, functions $\alpha \in L^{\infty}(\Omega), \beta \in L^{\infty}(\Sigma)$ are given.

Now the choice $D=\Sigma$ fits into our setting. It is known that a parabolic differential equation as in (P2) admits for each $u \in L^{2}(\Sigma)$ a unique weak solution $y \in W(0, T)$ with $W(0, T)=\left\{y \in L^{2}\left(0, T ; H^{1}(\Omega)\right) \mid y_{t} \in L^{2}\left(0, T ; H^{1}(\Omega)^{\prime}\right)\right\}$. Therefore, the mapping $S: u \mapsto \alpha y(T)$ is well defined, linear and compact from $L^{2}(\Sigma)$ to $H=L^{2}(\Omega)$. The operator $G$ is defined by $G: u \mapsto \tau y$, where $\tau: W(0, T) \rightarrow L^{2}(\Sigma)$ denotes the trace operator. Moreover, $G$ is linear and compact in $L^{2}(\Sigma)$ and thus $S$ and $G$ fulfill the assumptions in the definition of our general problem.

The corresponding regularized problem again looks almost the same except the first inequality constraint, which now is given by

$$
y(x, t) \geq y_{c}(x, t)-\varepsilon u(x) \quad \text { a.e. on } \Sigma .
$$


Thus, also this parabolic problem fits into the settings of our general optimization problem and can be solved by using regularized state constraints.

4. Existence of regular Lagrange multipliers. The regularity of the Lagrange multipliers associated with $\left(\mathrm{P}_{\varepsilon}\right)$ has already been discussed in [10] for slightly different types of mixed control-state constraints. There, constraints of the form $y \geq y_{c}+u$ were analysed. However, the results can be applied here, too, by substituting $u:=\varepsilon u$. For convenience of the reader, we shortly recall the main ideas.

We introduce the Lagrange functional $L=L(u, \mu, \lambda): L^{2}(D)^{3} \rightarrow \mathbb{R}$, by

$$
L(u, \mu, \lambda)=f(u)+\left(-\varepsilon u-G u+y_{c}, \mu\right)-(u, \lambda)
$$

with Lagrange multipliers $\mu, \lambda \in L^{2}(D)$. In all what follows, we will combine them to the vector of Lagrange multipliers $\omega:=(\mu, \lambda)$.

Definition. Let $u_{\varepsilon}$ be a solution of the problem $\left(\mathrm{P}_{\varepsilon}\right)$. Functions $\mu_{\varepsilon}, \lambda_{\varepsilon} \in L^{2}(D)$ are said to be regular Lagrange multipliers associated with $u_{\varepsilon}$, if the following relations are satisfied:

$$
\frac{\partial L}{\partial u}\left(u_{\varepsilon}, \mu_{\varepsilon}, \lambda_{\varepsilon}\right)=0
$$

and

$$
\begin{aligned}
\left(-\varepsilon u_{\varepsilon}-G u_{\varepsilon}+y_{c}, \mu_{\varepsilon}\right) & =0, & \mu_{\varepsilon} \geq 0 \\
\left(u_{\varepsilon}, \lambda_{\varepsilon}\right) & =0, & \lambda_{\varepsilon} \geq 0 .
\end{aligned}
$$

It is easy to verify that $\left(\mathrm{P}_{\varepsilon}\right)$, also called primal problem, can be equivalently expressed by

$$
\left(\mathrm{PP}_{\varepsilon}\right) \quad \inf _{u \in L^{2}(D)}\left\{\sup _{\omega \in L^{2}(D)^{2}, \omega \geq 0} L(u, \omega)\right\}
$$

where $\omega \geq 0$ means $\mu(x) \geq 0$ and $\lambda(x) \geq 0$ almost everywhere on $D$.

Thanks to the strict convexity of $f$, the primal problem admits a unique optimal solution $u_{\varepsilon}$ for every $\varepsilon>0$.

The Lagrange dual problem is defined by reversing the order of inf and sup,

$$
\left(\mathrm{DP}_{\varepsilon}\right) \quad \sup _{\omega \in L^{2}(D)^{2}, \omega \geq 0}\left\{\inf _{u \in L^{2}(D)} L(u, \omega)\right\} .
$$

If one can show that the optimal values of the primal and dual problem coincide and regular functions $\mu_{\varepsilon}$ and $\lambda_{\varepsilon}$ exist, solving the dual problem $\left(\mathrm{DP}_{\varepsilon}\right)$, then these functions represent Lagrange multipliers in the sense of the definition above (cf. [10], section 2). With the help of a separation theorem, it is shown in [10] that the optimal values of the primal and dual problem indeed agree, i.e., that there is no duality gap. Thus

$$
f\left(u_{\varepsilon}\right)=\sup \left(\mathrm{DP}_{\varepsilon}\right) .
$$

It remains to prove the existence of the Lagrange multipliers in $L^{2}(D)$. For this purpose we define the dual objective function by

$$
g(\mu, \lambda)=\left(y_{c}, \mu\right)-\frac{1}{2}\left\|a-\varepsilon \mu-G^{\star} \mu-\lambda\right\|_{\Lambda}^{2},
$$


where the norm $\|\cdot\|_{\Lambda}$ is given by $\Lambda=\kappa I+S^{\star} S$ and

$$
\|d\|_{\Lambda}^{2}=\left(d, \Lambda^{-1} d\right) .
$$

In [10] it is shown that the dual problem $\left(\mathrm{DP}_{\varepsilon}\right)$ can be expressed with the help of the dual objective function by

$$
\sup _{\omega \in L^{2}(D)^{2}, \omega \geq 0} g(\omega)=\sup _{\omega \in L^{2}(D)^{2}, \omega \geq 0}\left\{\inf _{u \in L^{2}(D)} L(u, \omega)\right\} .
$$

To be consistent with [10], we denote the negative objective functional $-g$ of $\left(\mathrm{DP}_{\varepsilon}\right)$ by $\varphi$

$$
\varphi(\omega)=-\left(y_{c}, \mu\right)+\frac{1}{2}\left\|a-\varepsilon \mu-G^{\star} \mu-\lambda\right\|_{\Lambda}^{2} .
$$

It is obvious that the dual problem is equivalent to minimizing $\varphi$ on the set of nonnegative functions of $L^{2}(D)^{2}$,

$$
\inf _{\omega \geq 0}\left\{-\left(y_{c}, \mu\right)+\frac{1}{2}\left\|a-\varepsilon \mu-G^{\star} \mu-\lambda\right\|_{\Lambda}^{2}\right\} .
$$

Hence, if there is an optimal $\omega_{\varepsilon}:=\left(\mu_{\varepsilon}, \lambda_{\varepsilon}\right) \in L^{2}(D)^{2}$ with $\omega_{\varepsilon} \geq 0$ minimizing $\varphi$, then $\mu_{\varepsilon}$ and $\lambda_{\varepsilon}$ are the Lagrange multipliers associated with the primal problem because of (4.1).

THEOREM 4.1. Assume that $G$ satisfies the following assumptions of boundedness

$$
\|G \mu\| \leq c_{G}\|\mu\| \quad \forall \mu \in L^{2}(D)
$$

and non-negativity, i.e.,

$$
u(x) \geq 0 \text { a.e. on } D \Rightarrow(G u)(x) \geq 0 \text { a.e. on } D \text {. }
$$

Then Lagrange multipliers $\mu_{\varepsilon}$ and $\lambda_{\varepsilon}$ associated with $\left(P_{\varepsilon}\right)$ exist in $L^{2}(D)$.

Proof: Let $\left\{\omega_{n}\right\}=\left\{\left(\mu_{n}, \lambda_{n}\right)\right\}$ be a minimizing sequence, i.e.

$$
\lim _{n \rightarrow \infty} \varphi\left(\omega_{n}\right)=j,
$$

where $j$ denotes the infimum of $\varphi$. First we have to show that this infimum exists. The $\Lambda$-norm in $\varphi$ can be estimated by the $L^{2}(D)$-norm, since the operator $\Lambda=\kappa I+S^{\star} S$ is positive definite and bounded, i.e. $\|\Lambda d\| \leq c_{\Lambda}\|d\|$ for all $d \in L^{2}(D)$. This implies

$$
\|d\|_{\Lambda}^{2}=\left(d, \Lambda^{-1} d\right)=\left(\Lambda\left(\Lambda^{-1} d\right), \Lambda^{-1} d\right) \geq \kappa\left\|\Lambda^{-1} d\right\|^{2} \geq \kappa c_{\Lambda}^{-2}\|d\|^{2}
$$

for all $d \in L^{2}(D)$. Therefore we will estimate the $\Lambda$-norm in (4.2) by

$$
\begin{aligned}
\left\|a-\varepsilon \mu-G^{\star} \mu-\lambda\right\|_{\Lambda}^{2} & \geq c_{1}\left\|a-\mu_{n}-G^{\star} \mu_{n}-\lambda_{n}\right\|^{2} \\
& \geq c_{1}\left(\|a\|^{2}-2\|a\|\left\|\mu_{n}+G^{\star} \mu_{n}+\lambda_{n}\right\|+\left\|\mu_{n}+G^{\star} \mu_{n}+\lambda_{n}\right\|^{2}\right) \\
& \geq c_{1}\left\|\mu_{n}+G^{\star} \mu_{n}+\lambda_{n}\right\|^{2}-c_{2}\left\|\mu_{n}+G^{\star} \mu_{n}+\lambda_{n}\right\|+c_{3},
\end{aligned}
$$

with certain positive constants $c_{i}, i=1,2,3$. For the first term, the non-negativity of $G$ implies $\left\|\mu_{n}+G^{\star} \mu_{n}+\lambda_{n}\right\|^{2} \geq\left\|\mu_{n}+\lambda_{n}\right\|^{2}$. Furthermore, we have $\left\|\mu_{n}+\lambda_{n}\right\|^{2}=$ 
$\left\|\mu_{n}\right\|^{2}+2\left(\mu_{n}, \lambda_{n}\right)+\left\|\lambda_{n}\right\|^{2} \geq\left\|\mu_{n}\right\|^{2}+\left\|\lambda_{n}\right\|^{2}$, since $\mu_{n}, \lambda_{n} \geq 0$. Together with the bondedness of $G$, this yields

$$
\left\|a-\varepsilon \mu-G^{\star} \mu-\lambda\right\|_{\Lambda}^{2} \geq c_{1}\left(\left\|\mu_{n}\right\|^{2}+\left\|\lambda_{n}\right\|^{2}\right)-c_{2}\left(1+c_{G}\right)\left\|\mu_{n}\right\|-c_{2}\left\|\lambda_{n}\right\|+c_{3} .
$$

Furthermore, $\left(y_{c}, \mu_{n}\right) \leq\left\|y_{c}\right\|\left\|\mu_{n}\right\|=c_{c}\left\|\mu_{n}\right\|, c_{c} \geq 0$, since $y_{c} \in L^{2}(D)$. Alltogether we obtain for $\varphi$

$$
\varphi\left(\omega_{n}\right) \geq c_{1}\left(\left\|\mu_{n}\right\|^{2}+\left\|\lambda_{n}\right\|^{2}\right)-c_{2}\left(\left\|\mu_{n}\right\|+\left\|\lambda_{n}\right\|\right)+c_{3},
$$

with some positive generic constants $c_{1}, c_{2}, c_{3}$. Thanks to the positive quadratic terms, $\varphi$ is bounded from below and the infimum exists. Additionally, (4.3) means that $\left\|\mu_{n}\right\|$ and $\left\|\lambda_{n}\right\|$ are bounded, otherwise $\varphi$ would tend to infinity. Consequently we can select weakly converging subsequences, w.l.o.g. $\mu_{n} \rightarrow \mu_{\varepsilon}$ and $\nu_{n} \rightarrow \bar{\nu}$. The weak lower semicontinuity of the objective functional $\varphi$ implies $j \geq \varphi\left(\omega_{\varepsilon}\right)$ giving in turn $j=\varphi\left(\omega_{\varepsilon}\right)$. The set $M=\left\{v(x) \in L^{2}(D) \mid v(x) \geq 0\right.$ a.e. in $\left.D\right\}$ is weakly closed in $L^{2}(D)$. Therefore, we have $\omega_{\varepsilon} \geq 0$ and consequently $\omega_{\varepsilon}$ is an optimal solution of the dual problem. Due to the strict convexity of $\varphi$, the optimal solution $\omega_{\varepsilon}$, i.e. the vector of Lagrange multipliers, is unique.

5. Pass to the limit. Given $\varepsilon>0$ we denote the unique solution of $\left(\mathrm{P}_{\varepsilon}\right)$ by $u_{\varepsilon}$ and the unique solution of $(\mathrm{P})$ by $\bar{u}$. Our next aim is to show the strong convergence of $u_{\varepsilon}$ to $\bar{u}$ as $\varepsilon \downarrow 0$.

Let $\left\{\varepsilon_{n}\right\}$ be a sequence of positive numbers tending to zero and let $\left\{u_{n}\right\}$ be the sequence of associated optimal solutions of $\left(\mathrm{P}_{\varepsilon_{n}}\right)$. The regularization term $\frac{\kappa}{2}\|u\|^{2}$ within the objective functional ensures that $\|u\|$ is bounded in $L^{2}(D)$. Thus we can select a weakly converging subsequence, $u_{n_{k}} \rightarrow \tilde{u}$. Everything what follows is also valid for any other weakly converging subsequence. Thus, a known argument yields that w.l.o.g. $u_{n} \rightarrow \tilde{u}$.

First we will show that $\tilde{u}=\bar{u}$. The main idea to prove this claim is the equality $f(\bar{u})=f(\tilde{u})$, that will be shown by the feasibility of $\tilde{u}$ for $(\mathrm{P})$ and $\bar{u}$ for $\left(\mathrm{P}_{\varepsilon}\right)$.

Lemma 5.1. Let $\tilde{u}$ be the weak limit of $\left\{u_{n}\right\}$. Then $\tilde{u}$ is feasible for $(\mathrm{P})$.

Proof: For every $\varepsilon_{n}>0$, the corresponding $u_{n}$ fulfills the constraint inequality

$$
\left(G u_{n}\right)(x) \geq y_{c}(x)-\varepsilon_{n} u_{n}(x) \quad \text { a.e. on } D \text {. }
$$

Moreover, $\varepsilon_{n}\left\|u_{n}\right\|$ converges to zero when $\varepsilon_{n} \downarrow 0$, due to the boundedness of $\left\|u_{n}\right\|$. Thus we can select a subsequence, w.l.o.g. $\left\{\varepsilon_{n} u_{n}\right\}$, converging to zero almost everywhere in $D$,

$$
\lim _{n \rightarrow \infty} \varepsilon_{n} u_{n}(x)=0 \quad \text { a.e. in } D \text {. }
$$

Furthermore, $\left\{G u_{n}\right\}$ converges strongly in $L^{2}(D)$ due to the compactness of $G$ and the weak convergence of $\left\{u_{n}\right\}$. Thus

$$
\lim _{n \rightarrow \infty} G u_{n}=G \tilde{u} \quad \text { for } u_{n} \rightarrow \tilde{u} .
$$

Therefore, passing to the limit $n \rightarrow \infty$, the first inequality constraint in $(\mathrm{P})$ is satisfied,

$$
(G \tilde{u})(x) \geq y_{c}(x) \quad \text { a.e. in } D \text {. }
$$


Since the set $M=\left\{u(x) \in L^{2}(D) \mid u(x) \geq 0\right.$ a.e. in $\left.D\right\}$ is weakly closed in $L^{2}(D)$ and $u_{n} \rightarrow \tilde{u}, u_{n} \geq 0 \forall n$, the second inequality constraint in $(\mathrm{P})$,

$$
\tilde{u}(x) \geq 0 \text { a.e. in } D
$$

is also satisfied. Thus $\tilde{u}$ is feasible for $(\mathrm{P})$.

Lemma 5.2. For every $\varepsilon>0$, the solution $\bar{u}$ of $(\mathrm{P})$ is feasible for $\left(\mathrm{P}_{\varepsilon}\right)$.

Proof: Clearly, $\bar{u}$ is feasible for $(\mathrm{P})$, hence the inequality constraints of $(\mathrm{P})$ are satisfied. Moreover, by $\bar{u}(x) \geq 0$ a.e. in $D$,

$$
(G \bar{u})(x) \geq y_{c}(x) \geq y_{c}(x)-\varepsilon \bar{u}(x) \quad \text { a.e. in } D
$$

holds true for every $\varepsilon>0$. Therefore $\bar{u}$ also fulfills the constraints of $\left(\mathrm{P}_{\varepsilon}\right)$.

THEOREM 5.3. The sequence of optimal solutions $\left\{u_{n}\right\}$ of $\left(\mathrm{P}_{\varepsilon_{n}}\right)$ converges strongly in $L^{2}(D)$ to $\bar{u}$, as $n \rightarrow \infty$, i.e $u_{n} \rightarrow \bar{u}$.

Proof: The feasibility of $\bar{u}$ and the optimality of $u_{n}$ for $\left(\mathrm{P}_{\varepsilon_{n}}\right)$ imply $f\left(u_{n}\right) \leq f(\bar{u})$ for all $\varepsilon_{n}$. Passing to the limit, one obtains

$$
f(\bar{u}) \geq \limsup _{n \rightarrow \infty} f\left(u_{n}\right) \geq \liminf _{n \rightarrow \infty} f\left(u_{n}\right) \geq f(\tilde{u}) \geq f(\bar{u}),
$$

since $f$ is weakly lower semicontinuous. For the last inequality in (5.1), we used the feasibilty of $\tilde{u}$ and the optimality of $\bar{u}$ for $(\mathrm{P})$. Thus we get $f(\tilde{u})=f(\bar{u})$ and the strict convexity of $f$ implies

$$
\tilde{u}=\bar{u}
$$

and hence $u_{n} \rightarrow \bar{u}$.

It remains to show that the convergence of $\left\{u_{n}\right\}$ is also strong, i.e. $u_{n} \rightarrow \bar{u}$. We argue as follows: From (5.1), the convergence

$$
\lim _{n \rightarrow \infty} f\left(u_{n}\right)=f(\bar{u})
$$

is obtained. By definition of $f$ we have

$$
\left\|u_{n}\right\|^{2}=\frac{2}{\kappa}\left(f\left(u_{n}\right)-\frac{1}{2}\left\|S u_{n}\right\|_{H}^{2}-\left(a, u_{n}\right)\right) .
$$

Since $S$ is a compact operator, the right hand side converges to the value at $\bar{u}$. Thus we have

$$
\lim _{n \rightarrow \infty}\left\|u_{n}\right\|^{2}=\frac{2}{\kappa}\left(f(\bar{u})-\frac{1}{2}\|S \bar{u}\|_{H}^{2}-(a, \bar{u})\right)=\|\bar{u}\|^{2} .
$$

As a well known fact, weak and norm convergence together yield strong convergence, i.e. $u_{n} \rightarrow \bar{u}$.

REMARK 5.4. Clearly, the state $y_{n}=S u_{n}$ converges strongly in $L^{2}(D)$ to $\bar{y}=S \bar{u}$, too. 
6. An optimization problem without control constraints. We will also analyze the following optimization problem:

$$
\left\{\begin{aligned}
\operatorname{minimize} & f(u)=\frac{1}{2}\|S u\|_{H}^{2}+\frac{\kappa}{2}\|u\|^{2}+(a, u) \\
\text { subject to } & (G u)(x) \geq y_{c}(x) \quad \text { a.e. in } D,
\end{aligned}\right.
$$

where the control constraint occuring in $(\mathrm{P})$ no longer appears. We impose the same assumptions on $D, S, G, a, y_{c}$ and $\kappa$ as for $(\mathrm{P})$. The corresponding regularized optimization problem now admits the form

$$
\left(\mathrm{Q}_{\varepsilon}\right) \quad\left\{\begin{aligned}
\text { minimize } & f(u) \\
\text { subject to } & (G u)(x) \geq y_{c}(x)-\varepsilon u(x) \quad \text { a.e. in } D .
\end{aligned}\right.
$$

The existence of an associated regular Lagrange multiplier $\mu_{\varepsilon}$ can be proven in a direct and rather trivial way. We substitute $v=\varepsilon u+G u$ and assume that $\varepsilon I+G$ has a continuous inverse operator in $L^{2}(D)$. If $G$ is positive semidefinit, then $\varepsilon I+G$ is invertible for all $\varepsilon>0$. Then we have $u=(\varepsilon I+G)^{-1} v=R v$, if we set $R=(\varepsilon I+G)^{-1}$. Define $\tilde{f}(v)=f(R v)$. In this way, the problem $\left(\mathrm{Q}_{\varepsilon}\right)$ becomes equivalent to

$$
\left(\tilde{\mathrm{Q}}_{\varepsilon}\right) \quad\left\{\begin{aligned}
\text { minimize } & \tilde{f}(v) \\
\text { subject to } & v(x) \geq y_{c}(x) \quad \text { a.e. in } D .
\end{aligned}\right.
$$

This is a problem with simple box constraints instead of state constraints. Therefore, one obtains an associated Lagrange multiplier in $L^{2}(D)$ by standard methods, cf. [10], section 4.1.

Now let $\left\{\varepsilon_{n}\right\}$ again be a positive sequence converging to zero and $\left\{u_{n}\right\}$ be the sequence of optimal solutions of $\left(\mathrm{Q}_{\varepsilon_{n}}\right)$. As $\varepsilon_{n}>0,\left(\varepsilon_{n} I+G\right)^{-1}$ exists as a continuous operator. As above, we denote the unique optimal solution of (Q) by $\bar{u}$. The proof of strong convergence $u_{n} \rightarrow \bar{u}$ is basically along the lines of the preceding section. However, we used $\bar{u}(x) \geq 0$ a.e. in $D$ in the proof of Lemma 5.2. This is the keypoint, where the single control constraint influences the theory above. Our new proof of the corresponding Lemma is based upon the existence of a Slater point for (Q) and an assumption of lower boundedness of $\bar{u}$.

LEMмA 6.1. Assume that the unique solution of $(Q)$ is bounded from below, i.e. $\bar{u}(x) \geq \bar{c}$ holds a.e. in D. Furthermore, let a function $\hat{u}(x)$ exist such that $\hat{u}(x)>0$ and $(G \hat{u})(x) \geq \tau>0$ holds a.e. in D. Then for every $\delta>0$, a positive $\varepsilon_{\delta}$ exists such that $\bar{u}+\delta \hat{u}$ is feasible for $\left(\mathrm{Q}_{\varepsilon}\right)$ for every $\varepsilon$ with $\varepsilon \leq \varepsilon_{\delta}$.

Proof: Due to the linearity of $G$, we have

$$
(G(\bar{u}+\delta \hat{u}))(x)=(G \bar{u})(x)+\delta(G \hat{u})(x) \geq y_{c}(x)+\delta \tau>y_{c}(x) \quad \text { a.e. in } D .
$$

Thus $\bar{u}+\delta \hat{u}$ satisfies the constraint of $(\mathrm{Q})$. For the constraint in $\left(\mathrm{Q}_{\varepsilon}\right)$, we obtain

$$
\begin{aligned}
(G(\bar{u}+\delta \hat{u})+\varepsilon(\bar{u}+\delta \hat{u}))(x) & =(G \bar{u})(x)+\varepsilon \bar{u}(x)+\delta((G \hat{u})(x)+\varepsilon \hat{u}(x)) \\
& \geq y_{c}(x)-\varepsilon|\bar{c}|+\delta \tau,
\end{aligned}
$$

since $\hat{u}(x) \geq 0$ holds a.e. in $D$. To make $\bar{u}+\delta \hat{u}$ feasible for $\left(\mathrm{Q}_{\varepsilon}\right), \delta \tau-\varepsilon|\bar{c}|$ has to be positive. This takes place for

$$
0<\varepsilon \leq \frac{\tau}{|\bar{c}|} \delta
$$


Therefore, $\varepsilon_{\delta}$ is defined by $\varepsilon_{\delta}:=\frac{\tau}{|\bar{c}|} \delta$.

As above, we can select a weakly converging subsequence and by the same arguments, as all these subsequences tend to the same weak limit, we have $u_{n} \rightarrow \tilde{u}$, where $\tilde{u}$ again denotes the weak limit. Since $f$ is continuous, we have

$$
f(\bar{u}+\delta \hat{u}) \leq f(\bar{u})+\delta_{f}
$$

and $\delta \downarrow 0$ implies $\delta_{f} \downarrow 0$. Together with Lemma 6.1 and the lower semicontinuity of $f$, we obtain for a fixed $\delta$

$$
f(\bar{u})+\delta_{f} \geq f(\bar{u}+\delta \hat{u}) \geq \limsup _{n \rightarrow \infty} f\left(u_{n}\right) \geq \liminf _{n \rightarrow \infty} f\left(u_{n}\right) \geq f(\tilde{u}) \geq f(\bar{u}),
$$

where the feasibilty of $\tilde{u}$ and the optimality of $\bar{u}$ for (Q) were used for the last inequality. The feasibilty of $\tilde{u}$ for (Q) can be proven analogue to Lemma 5.1, since the non-negativity of $u$ was not required in this proof. Passing to the limit $\delta \downarrow 0$, we finally get $\lim _{n \rightarrow \infty} f\left(u_{n}\right)=f(\bar{u})$ and, due to the strict convexity of $f, \bar{u}=\tilde{u}$.

The remaining part of the theory is along the lines of the preceding section and we obtain an equivalent result:

TheOrem 6.2. Assume that $\bar{u}$ and $\hat{u}$ satisfy the assumption of Lemma 6.1. Then the sequence of optimal solutions associated with $\left(\mathrm{Q}_{\varepsilon_{n}}\right)$, denoted by $\left\{u_{n}\right\}$, converges strongly in $L^{2}(D)$ to $\bar{u}$ as $\varepsilon_{n} \downarrow 0$, i.e.

$$
u_{n} \rightarrow \bar{u}, n \rightarrow \infty
$$

7. Numerical tests. We have tested the regularization of pointwise state constraints by the following example without control constraints:

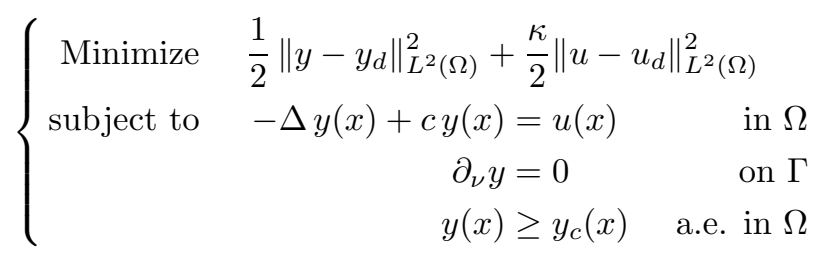

with $\Omega=[0,1] \times[0,1]$ and $\kappa \equiv c \equiv 1$. Analogous to (P1), one can easily verify that $(\mathrm{PT})$ fits into the settings of $(\mathrm{P})$.

Since $\Omega \subset \mathbb{R}^{2}$ and thus $\bar{y} \in H^{2}(\Omega) \subset C(\bar{\Omega})$, we consider the state constraint in $C(\bar{\Omega})$, where a Slater condition can be fulfilled. In this case, the Karush-Kuhn-Tucker theory ensures the existence of a Lagrange multiplier $\bar{\mu}$ in $C(\bar{\Omega})^{\prime}$. Hence, $\bar{\mu}$ in general represents a real Borel measure, cf. Casas [6]. By standard methods, the optimality system

$$
\begin{array}{rc}
-\Delta \bar{y}+c \bar{y}=\bar{u} & -\Delta \bar{p}+c \bar{p}=\bar{y}-y_{d}-\bar{\mu} \\
\partial_{\nu} \bar{y}=0 & \partial_{\nu} \bar{p}=0 \\
\bar{p}+\kappa\left(\bar{u}-u_{d}\right)=0 & \\
\bar{y}(x) \geq y_{c}(x) \quad, \quad \bar{\mu} \geq 0 \quad, \quad \int_{\Omega}\left(y-y_{c}\right) d \bar{\mu}=0
\end{array}
$$


is derived. The adjoint state $\bar{p}$ is the weak solution of the adjoint equation (7.1) and belongs to $W^{1, s}(\Omega)$ for all $1 \leq s \leq \frac{N}{N-1},[6]$.

The optimality system of the corresponding regularized problem $\left(\mathrm{PT}_{\varepsilon}\right)$ looks as follows:

$$
\begin{gathered}
-\Delta y_{\varepsilon}+c y_{\varepsilon}=u_{\varepsilon} \quad-\Delta p_{\varepsilon}+c p_{\varepsilon}=y_{\varepsilon}-y_{d}-\mu_{\varepsilon} \\
\partial_{\nu} y_{\varepsilon}=0 \\
\partial_{\varepsilon}=0 \\
p_{\varepsilon}+\kappa\left(u_{\varepsilon}-u_{d}\right)-\varepsilon \mu_{\varepsilon}=0 \\
y_{\varepsilon}(x) \geq y_{c}(x)-\varepsilon u_{\varepsilon}(x) \quad, \quad \mu_{\varepsilon}(x) \geq 0 \quad, \quad\left(y_{\varepsilon}-y_{c}+\varepsilon u_{\varepsilon}, \mu_{\varepsilon}\right)=0 .
\end{gathered}
$$

Now, in contrast to the case $\varepsilon=0$, the Lagrange multiplier $\mu_{\varepsilon}$ is a function of $L^{2}(\Omega)$. This regularity implies $p_{\varepsilon} \in H^{1}(\Omega)$.

The regularized optimization problem was solved numerically by a primal-dual active set strategy, see for instance [2] or [3]. We used a conform finite element method to solve the state equation and the adjoint equation. State and adjoint state were discretized by piecewise linear functions, whereas piecewise constant functions were applied to the control $u$ and the Lagrange multiplier $\mu$.

Two different examples were considered. The main difference between them is the regularity of the Lagrange multiplier. In the first example, we tested our regularization method on an exact solution with a continuous Lagrange multiplier, whereas the Lagrange multiplier in the second example represents a measure from $H^{1}(\Omega)^{\prime}$.

7.1. Example 1. This example is constructed such that the optimal state $\bar{y}$ and the adjoint state $\bar{p}$ are given by constant functions

$$
\bar{y}\left(x_{1}, x_{2}\right) \equiv \hat{y}, \quad \bar{p}\left(x_{1}, x_{2}\right) \equiv-c \kappa \hat{y},
$$

with a certain constant $\hat{y}$ and $c, \kappa$ as defined above. In our computations, we have chosen $\hat{y} \equiv 2$. Being constant, $\bar{y}$ and $\bar{p}$ fulfill the Neumann boundary condition as required in the state and the adjoint equation. From the state equation and the optimality condition we get

$$
\bar{u}=-\Delta \bar{y}+c \bar{y}=c \hat{y} \quad \text { and } \quad u_{d}=\bar{u}+\frac{1}{\kappa} \bar{p}=0 .
$$

The right hand side $y_{c}$ of the state constraint is defined by

$$
y_{c}\left(x_{1}, x_{2}\right)=\min \left(\hat{y}_{c}\left(x_{1}, x_{2}\right), \hat{y}\right)
$$

with $\hat{y}_{c}\left(x_{1}, x_{2}\right)=-20\left(x_{1}-0.5\right)^{2}-20\left(x_{2}-0.5\right)^{2}+\hat{y}+1$. Due to the complementary slackness condition, $\bar{\mu}$ has to vanish where $\bar{y}\left(x_{1}, x_{2}\right)=\hat{y}>y_{c}\left(x_{1}, x_{2}\right)$. Therefore, to make $\bar{y}, \bar{p}$ and $\bar{u}$ optimal, we choose for $\bar{\mu}$

$$
\bar{\mu}\left(x_{1}, x_{2}\right)=\max \left(\hat{y}_{c}\left(x_{1}, x_{2}\right)-\hat{y}, 0\right)
$$

This Lagrange multiplier is continuous, i.e. $\mu \in C(\bar{\Omega})$, and therefore the adjoint equation can be treated in a classical way. Finally, to fulfill all optimality conditions at $(\bar{y}, \bar{u}, \bar{p})$, we have to define

$$
y_{d}=\Delta \bar{p}-c \bar{p}+\bar{y}-\bar{\mu}=\left(1+c^{2} \kappa\right) \hat{y}-\bar{\mu} .
$$


The functions $y_{d}$ and $y_{c}$ are shown in figures 7.1 and 7.2.

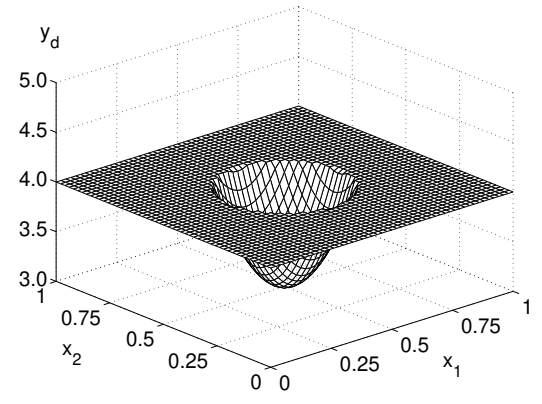

Fig. 7.1. Desired state $y_{d}$

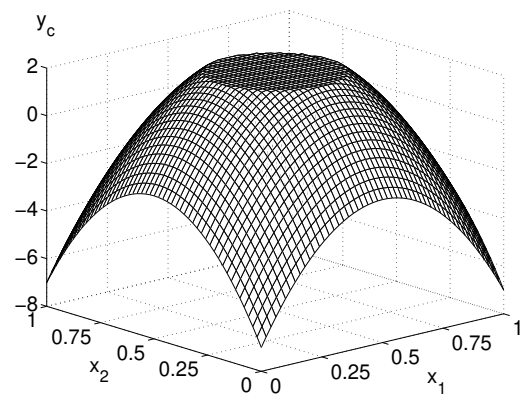

FIG. 7.2. Function $y_{c}$ in the state constraint

The following figures show the numerical solution of the regularized problem for $\varepsilon=0.008$. In all computations considered here, the mesh size was $h=0.04$. The numerical solutions of the regularized problem $\left(\mathrm{PT}_{\varepsilon}\right)$ are denoted by $(.)_{h}$. Notice that $\bar{u}\left(x_{1}, x_{2}\right) \equiv \bar{y}\left(x_{1}, x_{2}\right) \equiv 2$ and $\bar{p}\left(x_{1}, x_{2}\right) \equiv-2$.

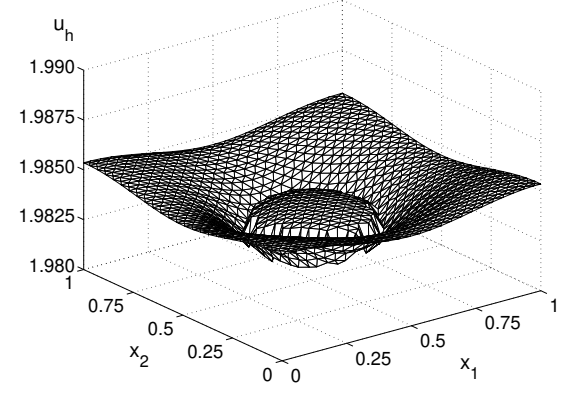

FIG. 7.3. Control $u_{h}$ at $\varepsilon=0.008$

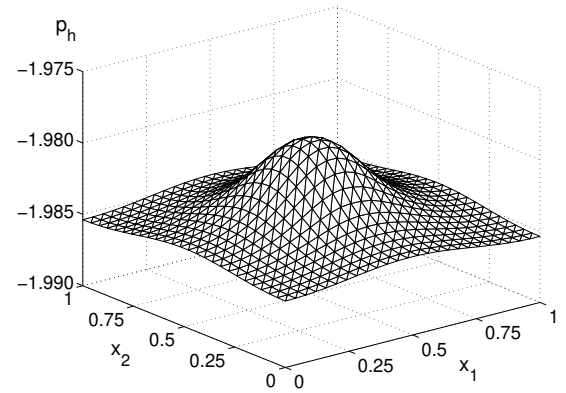

FIG. 7.5. Adjoint state $p_{h}$ at $\varepsilon=0.008$

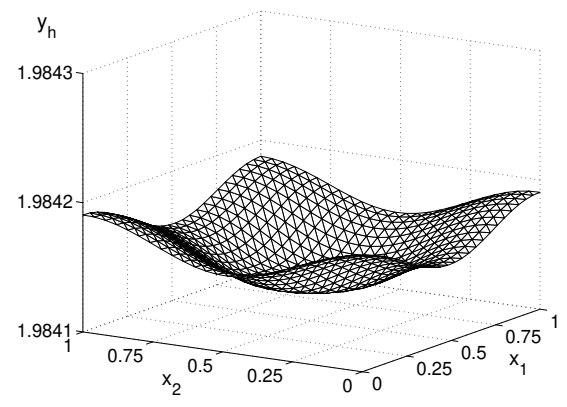

FIG. 7.4. State $y_{h}$ at $\varepsilon=0.008$

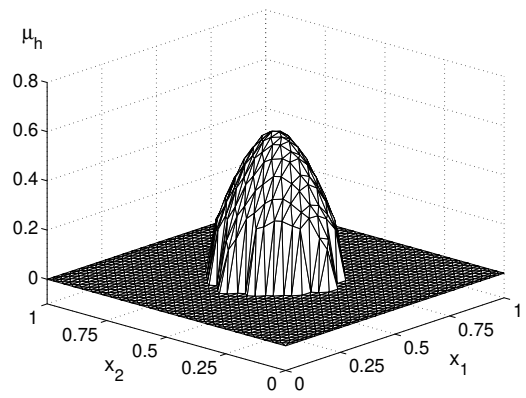

FIG. 7.6. Lagrange multiplier $\mu_{h}$ at $\varepsilon=0.008$

The behaviour of $u_{h}, y_{h}, p_{h}$ and $\mu_{h}$ for $\varepsilon \downarrow 0$ is of particular interest. The following figures illustrate the $L^{2}$-norms of the difference between the numerical solution of $\left(\mathrm{PT}_{\varepsilon}\right)$ and the exact solution of $(\mathrm{PT})$ for $\varepsilon=0.2,0.1,0.05,0.0025,0.00125$ and 
0.000625. The $L^{2}$-norms were approximated with third order Gaussian quadrature. The exact solution is indicated by the subscript "opt".

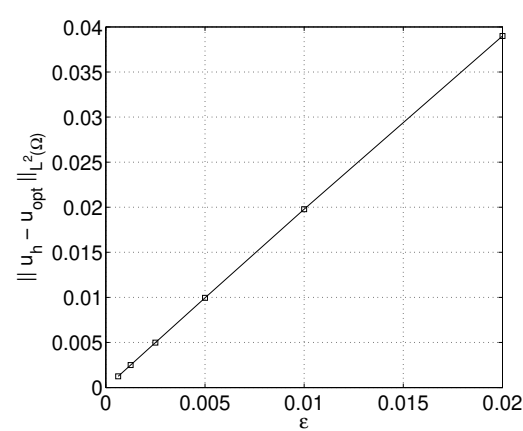

FIG. 7.7. $\left\|u_{h}-u_{o p t}\right\|$

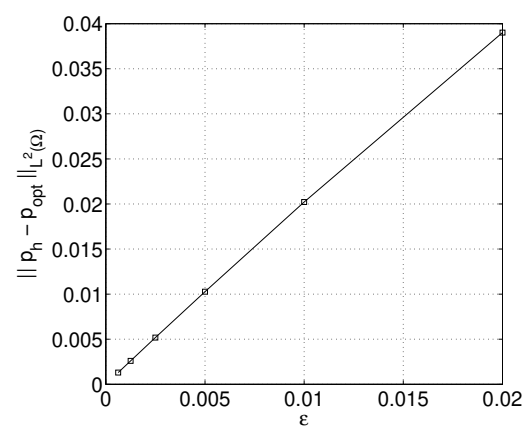

FIG. 7.9. $\left\|p_{h}-p_{\text {opt }}\right\|$

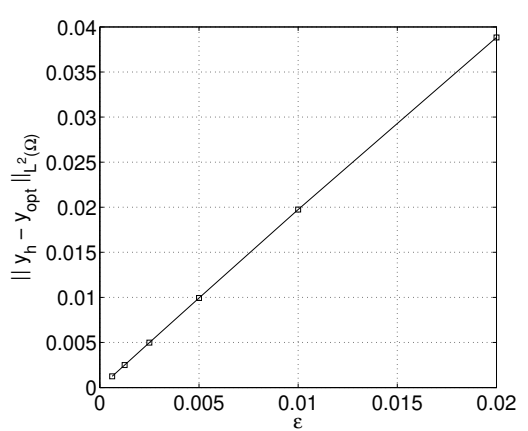

FIG. 7.8. $\left\|y_{h}-y_{o p t}\right\|$

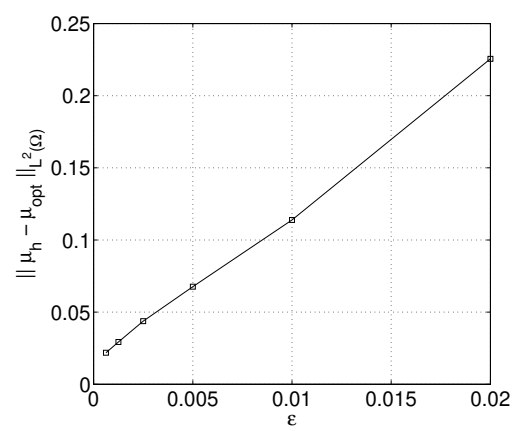

FIG. 7.10. $\left\|\mu_{h}-\mu_{o p t}\right\|$

7.2. Example 2. We now define the adjoint state $\bar{p}$ by

$$
\bar{p}\left(x_{1}, x_{2}\right)=\left\{\begin{array}{cl}
0.5-x_{1}^{2} & , x_{1}<0.5 \\
0.25 & , x_{1} \geq 0.5
\end{array}\right.
$$

It is easy to verify, that $\bar{p}$ satisfies the Neumann boundary condition. The function $\bar{p}$ belongs to $H^{1}(\Omega)$, hence we know $\Delta \bar{p} \in H^{1}(\Omega)^{\prime}$. Moreover, $\nabla \bar{p}$ exhibits a discontinuity at $x_{1}=0.5$. Therefore, $\bar{\mu}$ consists of a regular part in $\Omega \backslash\left\{\left(x_{1}, x_{2}\right) \mid x_{1}=0.5\right\}$ and a singular part (a $\delta$-distribution with respect to $x_{1}$ ) concentrated on $\hat{\Gamma}=\left\{\left(x_{1}, x_{2}\right) \in\right.$ $\left.\Omega \mid x_{1}=0.5\right\}$. We define the regular parts of $\bar{\mu}$ on $\Omega \backslash \hat{\Gamma}$ by

$$
\begin{aligned}
& \bar{\mu} \equiv 0 \text { in } \Omega_{1}=\left\{\left(x_{1}, x_{2}\right) \in \Omega \mid x_{1}<0.5\right\} \\
& \text { and } \\
& \bar{\mu} \equiv 1 \text { in } \Omega_{2}=\left\{\left(x_{1}, x_{2}\right) \in \Omega \mid x_{1}>0.5\right\}
\end{aligned}
$$

Again, the optimal state is defined by

$$
\bar{y} \equiv \hat{y} \equiv 2
$$


Due to the complementary slackness conditions, $\bar{y}$ and $y_{c}$ must satisfy $\bar{y} \geq y_{c}$ a.e. in $\Omega_{1}$ and $\bar{y}=y_{c}$ a.e. in $\Omega_{2}$. Hence, we define $y_{c}$ by

$$
y_{c}\left(x_{1}, x_{2}\right)= \begin{cases}2 x_{1}+\hat{y}-1, & x_{1}<0.5 \\ \hat{y} & , x_{1} \geq 0.5\end{cases}
$$

This function is also presented in figure 7.12. Since $\bar{p}$ is infinitely many times differentiable in $\Omega_{1}$ and $\Omega_{2}$, respectively, we can evaluate the adjoint equation $y_{d}=$ $\Delta \bar{p}-c \bar{p}+\hat{y}-\bar{\mu}$ separately in $\Omega_{1}$ and $\Omega_{2}$ to obtain the objective state function $y_{d}$. Thus, we get

$$
y_{d}\left(x_{1}, x_{2}\right)= \begin{cases}\hat{y}-2-0.5 c+c x_{1}^{2}, & x_{1}<0.5 \\ \hat{y}-1-0.25 c & , x_{1}>0.5 .\end{cases}
$$

Since $x_{1}=0.5$ belongs to the grid, we need discrete values of $y_{d}$ at $x_{1}=0.5$ for the numerical calculation. For convenience, we define

$$
y_{d}\left(x_{1}, x_{2}\right) \equiv \hat{y}-1.5-0.25 c \quad \text { on } \hat{\Gamma} .
$$

This value just represents the arithmetic mean of the functions for $y_{d}$ in $\Omega_{1}$ and $\Omega_{2}$. This function is shown in figure 7.11.

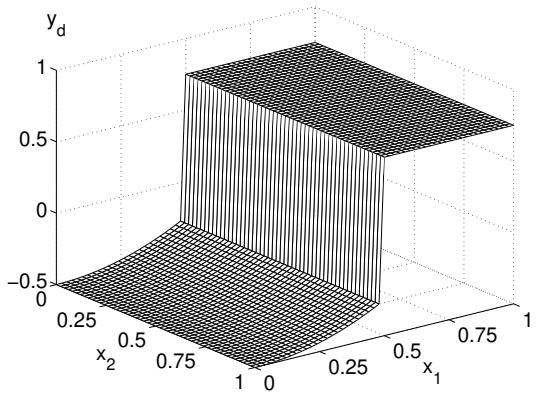

FIG. 7.11. Desired state $y_{d}$

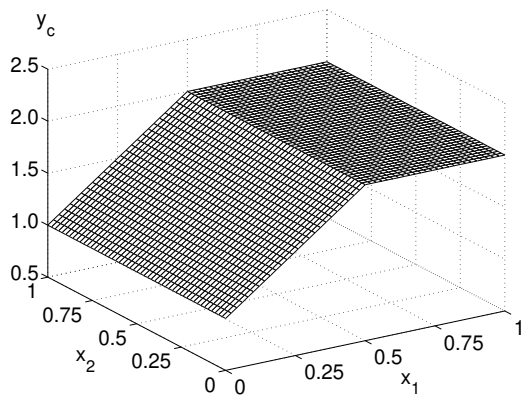

FIG. 7.12. Function $y_{c}$ in the state constraint

The optimal control can again be derived from the state equation. With $c=1$ we get

$$
\bar{u}\left(x_{1}, x_{2}\right) \equiv c \hat{y} \equiv 2 .
$$

It remains to define $u_{d}$, which is given by

$$
u_{d}=\bar{u}+\frac{1}{\kappa} \bar{p}= \begin{cases}\frac{1}{\kappa}\left(0.5-x_{1}^{2}\right)+c \hat{y}, & x_{1}<0.5 \\ 0.25+c \hat{y} & , x_{1} \geq 0.5 .\end{cases}
$$

We exemplarily present the numerical solution of the regularized problem for $\varepsilon=$ 0.002 . 


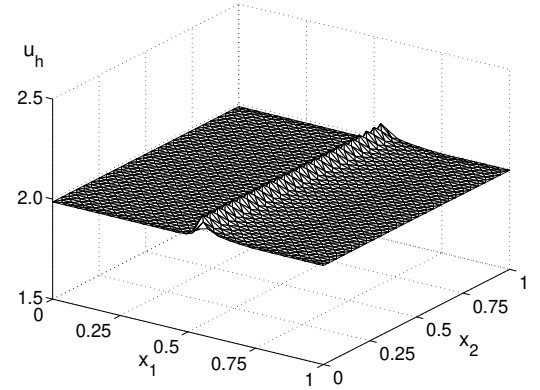

FIG. 7.13. Control $u_{h}$ at $\varepsilon=0.002$

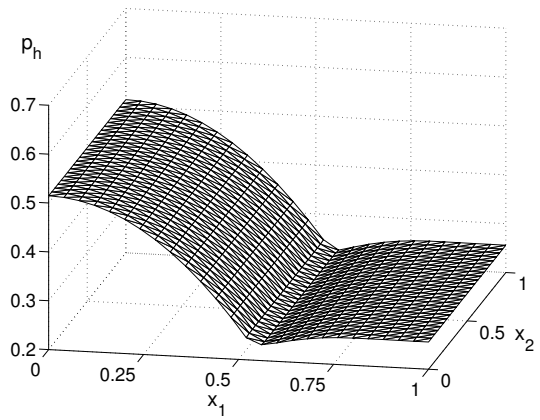

FIG. 7.15. Adjoint state $p_{h}$ at $\varepsilon=0.002$

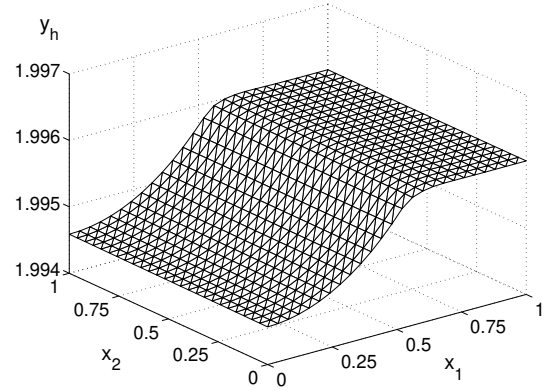

FIG. 7.14. State $y_{h}$ at $\varepsilon=0.002$

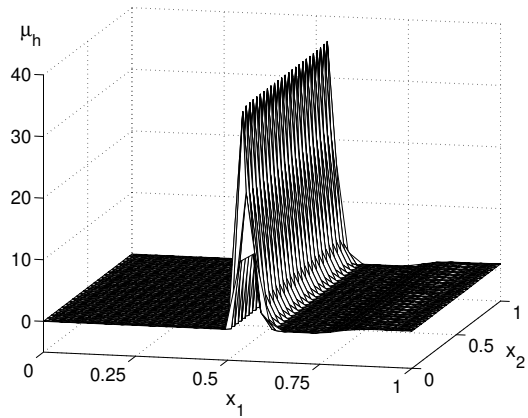

FIG. 7.16. Lagrange multiplier $\mu_{h}$ at $\varepsilon=0.002$

We observe that the Lagrange multiplier tends to a measure with singular part located at $\hat{\Gamma}$. The following figures show the convergence behaviour for $\left\|u_{h}-u_{o p t}\right\|,\left\|y_{h}-y_{\text {opt }}\right\|$ and $\left\|p_{h}-p_{\text {opt }}\right\|$.

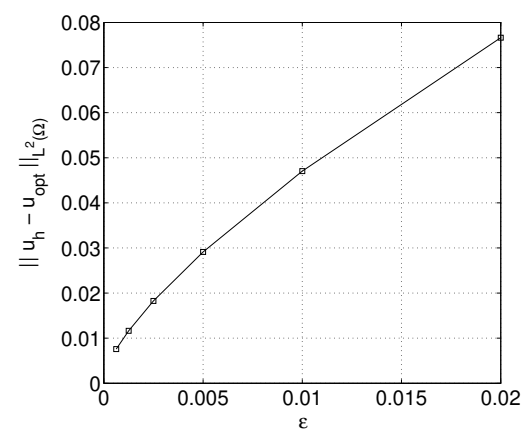

FIG. 7.17. $\left\|u_{h}-u_{\text {opt }}\right\|$

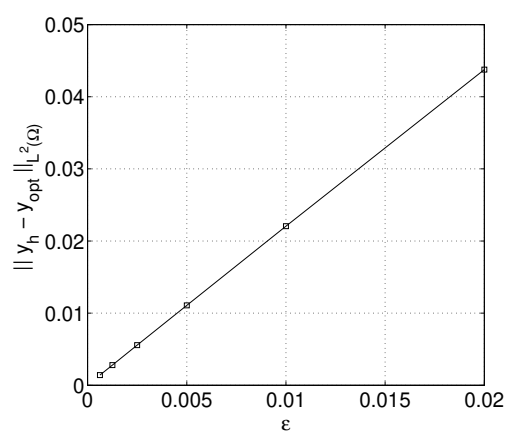

FIG. 7.18. $\left\|y_{h}-y_{o p t}\right\|$ 


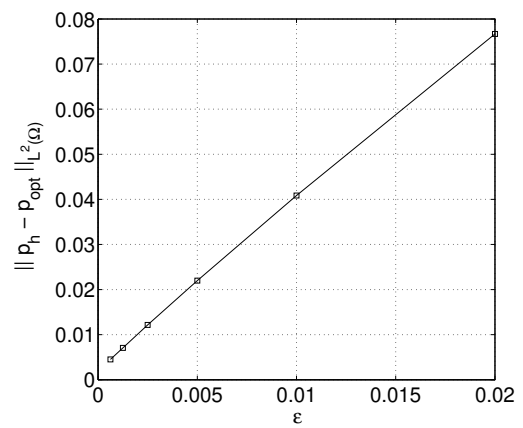

FIG. 7.19. $\left\|p_{h}-p_{\text {opt }}\right\|$

\section{REFERENCES}

[1] N. Arada and J.-P. Raymond. Optimal control problems with mixed control-state constraints. To appear.

[2] M. Bergounioux, K. Ito, and K. Kunisch. Primal-dual strategy for constrained optimal control problems. SIAM J. Control and Optimization, 37:1176-1194, 1999.

[3] M. Bergounioux and K. Kunisch. Primal-dual strategy for state-constrained optimal control problems. Computational Optimization and Applications, 22:193-224, 2002.

[4] M. Bergounioux and F. Tröltzsch. Optimal control of linear bottleneck problems. ESIAM, Control, Optimisation and Calculus of Variations, 3:235-250, 1998.

[5] M. Bergounioux and F. Tröltzsch. Optimal control of semilinear parabolic equations with stateconstraints of bottleneck type. ESIAM, Control, Optimisation and Calculus of Variations, 4:595-608, 1999.

[6] E. Casas. Boundary control of semilinear elliptic equations with pointwise state constraints. SIAM J. Control and Optimization, 31:993-1006, 1993.

[7] E. Casas, J. P. Raymond, and H. Zidani. Pontryagin's principle for local solutions of control problems with mixed control-state constraints. SIAM J. Control and Optimization, 39:1182-1203, 2000.

[8] K. Kunisch and A. Rösch. Primal-dual active set strategy for a general class of constrained optimal control problems. SIAM Journal Optimization, to appear.

[9] J. P. Raymond. Nonlinear boundary control of semilinear parabolic problems with pointwise state constraints. Discrete Contin. Dyn. Syst., 3:341-370, 1997.

[10] F. Tröltzsch. Regular Lagrange multipliers for control problems with mixed pointwise controlstate constraints. To appear. 